\title{
Extended family history of type 1 diabetes in HLA-predisposed children with and without islet autoantibodies
}

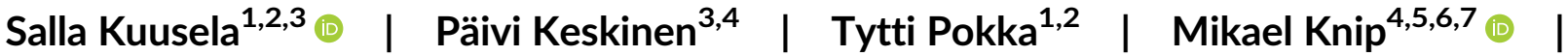 \\ Jorma llonen $^{8}$ | Paula Vähäsalo ${ }^{1,2}$ | Riitta Veijola ${ }^{1,2}$ (i)
}

${ }^{1}$ Department of Pediatrics, PEDEGO Research Unit, Medical Research Center, University of Oulu, Oulu, Finland

${ }^{2}$ Department for Children and Adolescents, Medical Research Center, Oulu University Hospital, Oulu, Finland

${ }^{3}$ Department for Children and Adolescents, Tampere University Hospital, Tampere, Finland

${ }^{4}$ Tampere Centre for Child Health Research, Tampere University Hospital, Tampere, Finland

${ }^{5}$ Children's Hospital, University of Helsinki and Helsinki University Hospital, Helsinki, Finland

${ }^{6}$ Research Programs for Clinical and Molecular Metabolism, Faculty of Medicine, University of Helsinki, Helsinki, Finland

${ }^{7}$ Folkhälsan Research Center, Helsinki, Finland ${ }^{8}$ Immunogenetics Laboratory, University of Turku, Turku, Finland

\section{Correspondence}

Riitta Veijola, MD, PhD, Department of Pediatrics, Medical Research Center Oulu, PO Box 5000, FIN-90014 University of Oulu, Oulu, Finland.

Email: riitta.veijola@oulu.fi

Funding information

Academy of Finland, Grant/Award Number: 292538; Centre of Excellence in Molecular Systems Immunology and Physiology Research, Grant/Award Number: 250114; Diabetes Research Foundation, Finland; European Commission, Grant/Award Number: BMH4-CT98-3314; Juvenile Diabetes Research Foundation International (JDRF), Grant/Award Numbers: 1-SRA-2016-342-MR, 1-SRA-2019-732-M-B; Novo Nordisk Fonden; Pediatric Research Foundation, Finland; Sigrid Juselius Foundation, Finland; Special Research Funds for University

\begin{abstract}
Objective: The aim of this study was to explore the extended family history of type 1 diabetes in children at genetic risk and define the impact of a positive family history on the development of islet autoimmunity and type 1 diabetes.

Methods: The subjects were participants in The Finnish Type 1 Diabetes Prediction and Prevention (DIPP) study and carried increased HLA-conferred risk for type 1 diabetes. The case children $(N=343)$ were positive for at least one islet autoantibody, and the control children ( $N=343$ ) matched by age, gender and class II HLA genotype were negative for islet autoantibodies at the time of data collection. Extended family history of type 1 diabetes was obtained by using a structured questionnaire.

Results: Among children who were autoantibody positive and progressed to type 1 diabetes $62.2 \%$ (28/45) had at least one relative with type 1 diabetes. Interestingly, $57.8 \%$ of these children $(26 / 45)$ had such a relative outside the nuclear family compared to $30.7 \%$ of children with no autoantibodies $(P=.001), 35.2 \%$ of those with only classical islet cell antibodies $(P=.006)$, and $35.2 \%$ of non-progressors with biochemical autoantibodies $(P=0.011)$. A positive history of type 1 diabetes in the paternal extended family was more common in children with multiple biochemical autoantibodies compared to those with only one biochemical autoantibody $(P=.010)$. No association between the specificity of the first appearing autoantibody and family history of the disease was found.
\end{abstract}

Conclusions: Type 1 diabetes in relatives outside the nuclear family is a significant risk factor for islet autoimmunity and progression to clinical disease in HLA susceptible children.

\section{KEYWORDS}

children, family history, genetic risk, HLA, islet autoimmunity, type 1 diabetes

Abbreviations: DIPP, The Finnish Type 1 Diabetes Prediction and Prevention study; FDR, first-degree relative; GADA, antibodies against glutamic acid decarboxylase; IAA, insulin autoantibody; IA-2A, antibodies against insulinoma-associated antigen-2; ICA, islet cell antibody; ZnT8A, antibodies against zinc transporter 8.

This is an open access article under the terms of the Creative Commons Attribution-NonCommercial-NoDerivs License, which permits use and distribution in any medium, provided the original work is properly cited, the use is non-commercial and no modifications or adaptations are made.

(C) 2020 The Authors. Pediatric Diabetes published by John Wiley \& Sons Ltd. 
Hospitals in Finland; The Alma and K.A. Snellman Foundation, Finland; The Finnish Medical Foundation

\section{1 | INTRODUCTION}

Type 1 diabetes is considered as an autoimmune disease caused by interaction between genetic susceptibility and environmental factors. Finland has the highest incidence of type 1 diabetes worldwide, and in 2006 the peak incidence of 64.9/100000/year in children under the age of 15 was reported. ${ }^{1}$

Type 1 diabetes has a subclinical phase identified by circulating islet autoantibodies. There is, however, wide individual variation in the duration of the progression from islet autoimmunity to clinical diabetes. ${ }^{2}$ Observations from prospective follow-up studies starting from birth have demonstrated that a substantial proportion of children who are genetically at risk and progress to type 1 diabetes have developed islet autoimmunity very early, between 9 months and 2 years of age..$^{3-6}$ It has also been observed that seroconversion occurs earlier and median autoantibody levels are higher in children who progress to clinical type 1 diabetes before puberty. ${ }^{7-10}$ In all, over $80 \%$ of children with multiple islet autoantibodies progress to symptomatic, insulin-requiring diabetes within 15 years after developing multiple islet autoantibodies, whereas only a minority of the children who remain positive for only a single islet autoantibody develops type 1 diabetes.

Parental type 1 diabetes increases the risk of the child to develop the disease. ${ }^{11-13}$ If the child has a father with type 1 diabetes, the disease risk is about two times higher when compared to a child who has a mother with type 1 diabetes (6\% vs 3\%). The younger the age at the onset of diabetes in the type 1 diabetic father the greater the risk of the disease is in his offspring. The risk of type 1 diabetes is also increased among the siblings of children with type 1 diabetes, and the magnitude of this risk is dependent on the siblings' class II HLA genotype. ${ }^{14}$ Risk for islet autoimmunity is dramatically higher in DR3/4 siblings who share both HLA haplotypes with their diabetic sibling compared to siblings who do not share both of these HLA haplotypes with the diabetic sibling. ${ }^{15,16}$ Less data is available on the role of a positive family history of type 1 diabetes in other family members than the father, mother or siblings, that is, in relatives outside the nuclear family.

The aim of this study was to investigate the prevalence of type 1 diabetes in the extended families of children at increased HLAconferred disease risk and participating in the prospective Type 1 Diabetes Prediction and Prevention (DIPP) study in Finland, and compare the development of islet autoimmunity and progression to type 1 diabetes in children with and without a positive family history of type 1 diabetes.

\section{2 | METHODS}

The Finnish DIPP study is a population-based prospective followup study which was launched in 1994 and recruits all children born in three University Hospitals in Finland (Oulu, Tampere and Turku) for screening of class II HLA-conferred genetic susceptibility for type 1 diabetes from cord blood. Children with eligible HLA genotypes associated with increased risk are invited to participate in follow-up until the age of 15 years with regular measurement of autoantibodies against islet cells (ICA), insulin (IAA), GAD65 (GADA), and islet antigen 2 (IA-2A) from serum samples taken at 3 to 12 months' intervals. ICA was used as the primary screening tool for islet autoimmunity until the end of 2002. If the child turned positive to ICA or progressed to type 1 diabetes, all samples taken during the follow-up were analyzed also for IAA, IA-2A and GADA. Children born since 2003 have been regularly analyzed for all four autoantibodies from all their samples. In addition, if the child was found to be positive for any of the four abovementioned autoantibodies, zinc transporter 8 antibodies (ZnT8A) have been measured from all samples of the child. Data on growth, nutrition, and general health-related events including possible medications has been recorded at the clinical study visits. In addition, information on family history of type 1 diabetes among firstdegree relatives (FDR, i.e. mother, father or sibling) has been recorded at the time of birth and updated during the follow-up if necessary.

\section{1 | Genetic screening}

HLA-conferred susceptibility to type 1 diabetes was screened centrally at University of Turku using cord blood. Sequence specific oligonucleotide probes specific for HLA-DQB1*02, DQB1*03:01, DQB1*03:02 and DQB1*06:02/3 alleles were used initially, and genotyping was step-wise expanded to cover more alleles in DQB1 and DQA1 loci as well as DRB1*04 subtypes. The detailed procedures have been described previously. ${ }^{5}$ The children in the current study population were categorized into six groups according to recent classification of HLA genotypes conferring high, moderate, slightly increased, neutral, or slightly decreased risk for type 1 diabetes, or protection for the disease according to Ilonen et al $2016 .{ }^{17}$

\section{2 | Autoantibody analyses}

Islet cell antibodies (ICA), insulin autoantibodies (IAA), antibodies against glutamic acid decarboxylase (GADA) and insulinoma antigen (IA-2A) were analyzed in the Diabetes Research Laboratory at the Department of Pediatrics, University of Oulu. Antibodies against zinc transporter 8 (ZnT8A) were analyzed in the PEDIA laboratory, University of Helsinki. DIPP participants who seroconverted to positivity for any of these autoantibodies (ICA, IAA, IA-2A, GADA or ZnT8A) were scheduled for follow-up visits 
at 3-month intervals. The age at seroconversion was defined as the age at which at least one of the islet autoantibodies was detected for the first time. The seroconversion to multiple autoantibody positivity was defined as the age when at least two biochemical (IAA, IA-2A, GADA or ZnT8A) autoantibodies were detected in the same sample for the first time.

\subsection{Study design and participants}

For the current analysis we invited a total of 343 DIPP families with a child positive for at least one islet autoantibody (case family) to answer the structured questionnaire of extended family history of type 1 diabetes. In addition, we invited control families with a DIPP child negative for islet autoantibodies and matched for date of birth, sex and HLA-risk category with the DIPP child in the case family. Qualification criteria for autoantibody negativity was that no positive results had been obtained in samples collected from birth until the date when extended family history data was collected. All 343 case families and the 343 matched control families were followed in the DIPP clinic at Oulu University Hospital and received information about this survey by a trained study nurse either at the DIPP visit or by an invitation letter. When informed consent was received, data about the extended family history of diabetes was collected between October 2003 and August 2008. The data was not obtained from 47 case families and 30 control families. Thus, our case control dataset included 296 and 313 children with and without islet autoantibodies, respectively (Figure 1). In the group of autoantibody positive children there were 177 children who were positive for ICA but had no other islet autoantibodies (ICA only).

Follow-up data until August 2016 was included (Figure 1). In the group of autoantibody negative children 22 developed ICA only during the follow-up, and another four children developed multiple biochemical islet autoantibodies, and one of them progressed to type 1 diabetes. His age at seroconversion was 4.1 years and age at diagnosis 7.7 years. At the end of the follow-up a total of 287 children remained autoantibody negative.

In the group of 296 autoantibody positive children, 177 were positive only for ICA at the time of the data collection, but 17 of them developed also biochemical islet autoantibodies during the follow-up. A total of 45 out of the 136 children with biochemical islet

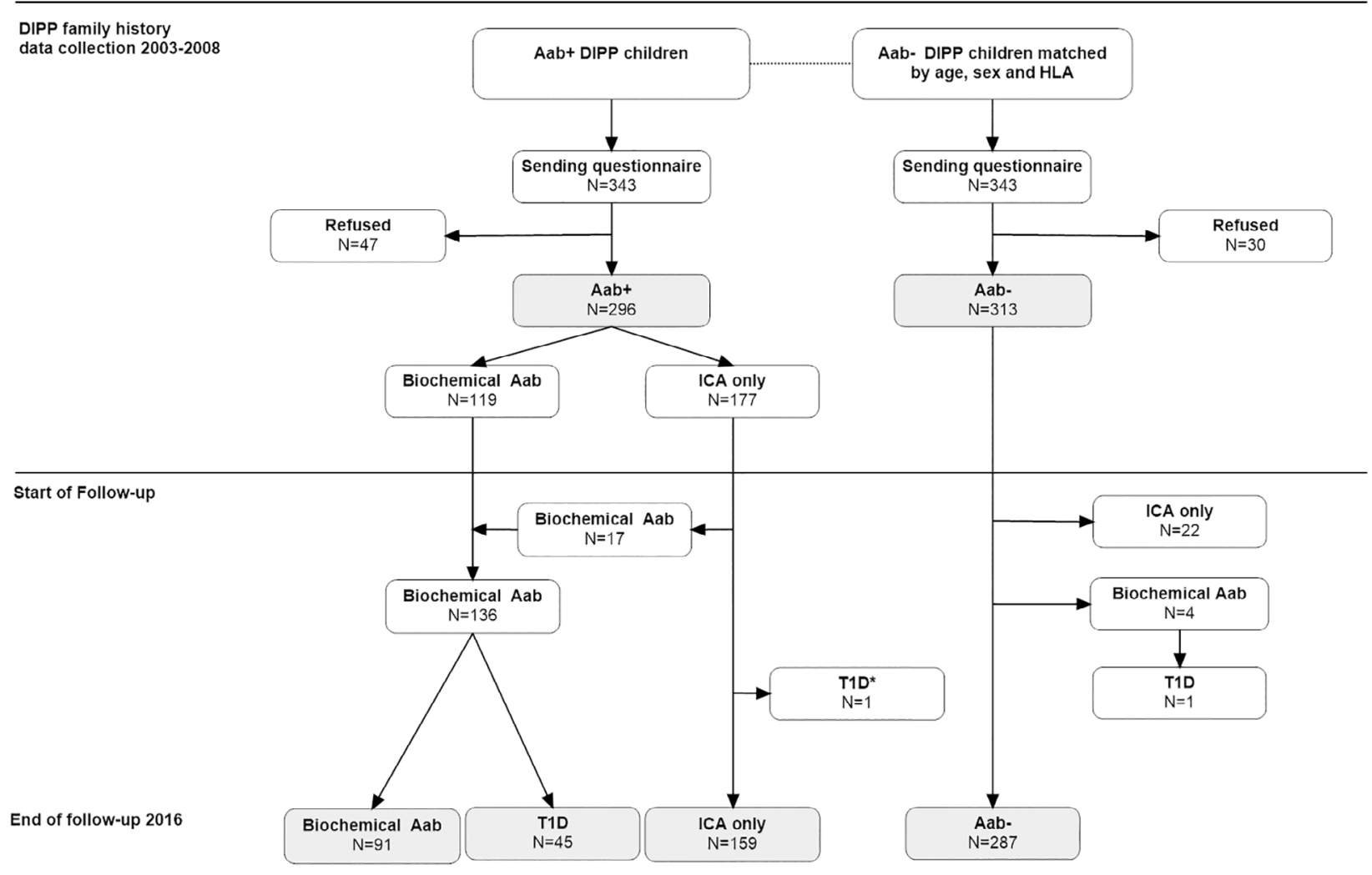

FIGURE 1 Description of the study population. Families participating in the Finnish Type 1 Diabetes Prediction and Prevention (DIPP) study were included. A total of 343 case families with a child positive for at least one islet autoantibody (Aab+) and 343 control families with an autoantibody negative (Aab-) child matched for date of birth, sex and class II HLA risk were invited to participate the data collection of extended family history of type 1 diabetes. At the end of follow-up the children were categorized into four groups: children remaining autoantibody negative (Aab-), children with only classical islet cell antibodies (ICA only), children with one or more biochemical autoantibodies (Biochemical Aab) and children who progressed to type 1 diabetes (T1D) 
autoantibodies developed type 1 diabetes by the end of the follow up. In addition, one study subject who was positive for ICA only progressed to type 1 diabetes at the age of 13 years. This child had also been diagnosed with steroid-resistant nephrotic syndrome and received intermittent prednisolone therapy from the age of 9 years and rituximab therapy from the age of 11 years. Because of the unusual disease history this child was not included in any of the final analyses (Figure 1).

TAB LE 1 Clinical characteristics of the study population according to autoantibody (Aab) groups defined after follow-up: autoantibody negative children, autoantibody positive children, children with islet cell antibodies only (ICA only), children with biochemical islet autoantibodies (biochemical Aab) and children who progressed to type 1 diabetes (T1D)

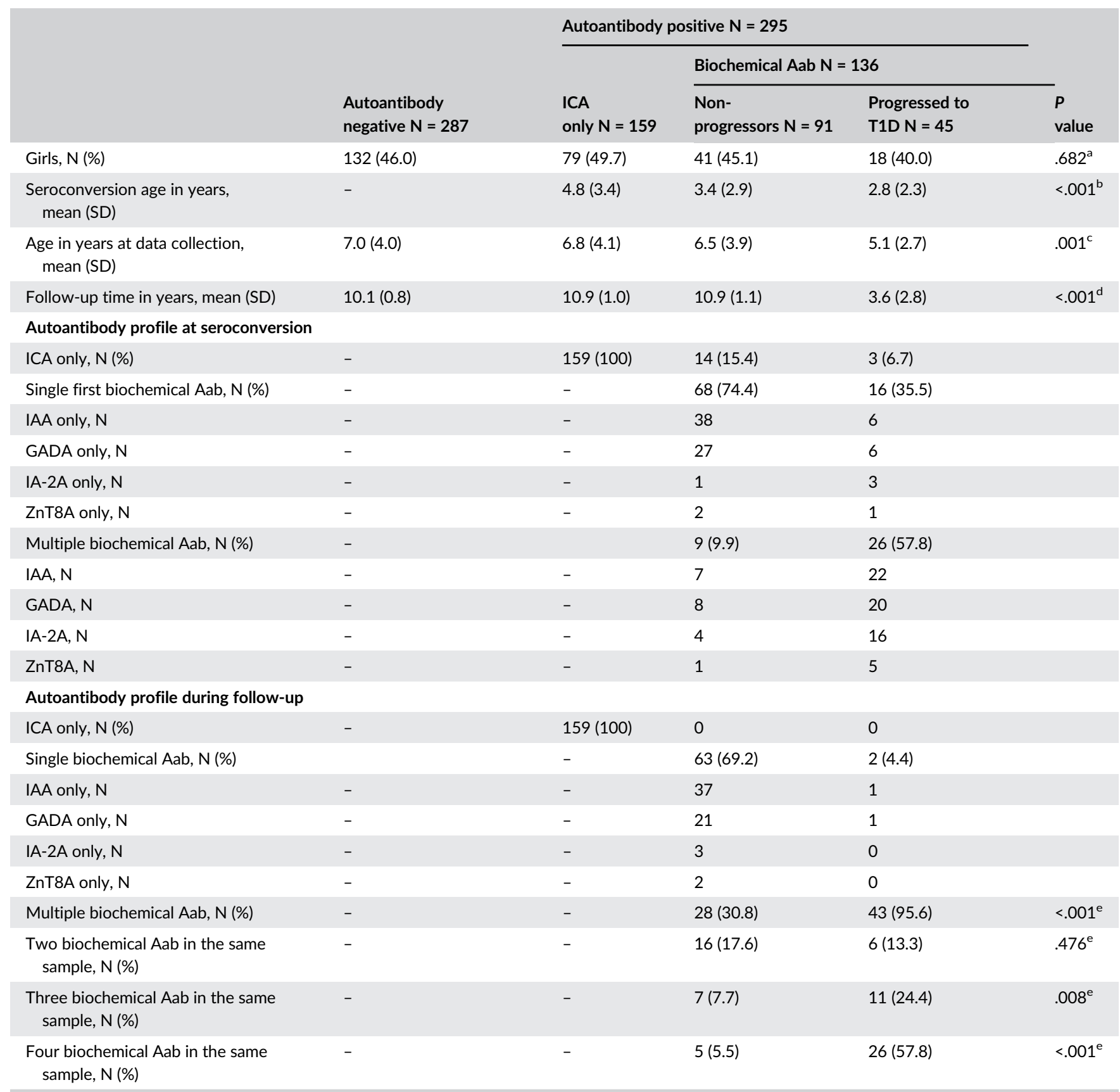

a'Exact Chi-square test.

${ }^{b}$ ANOVA: Welch test. Post hoc pairwise Games-Howell: ICA only vs non-progressors, $P=.002$; ICA only vs T1D, $P<.001$.

CANOVA: Welch test. Post hoc pairwise Games-Howell: Autoantibody negative vs T1D, $P<.001$; ICA only vs T1D, $P=.008$.

${ }^{\mathrm{d} A N O V A}$ : Welch test. Post hoc pairwise Games-Howell: T1D vs other groups, $P<.001$ in all comparisons.

eSND test. 
In the group of 295 children positive for any islet autoantibody the mean age at seroconversion was 4.1 years (SD 3.2; range 0.2-15.2 years). The children who developed biochemical autoantibodies and those who progressed to type 1 diabetes were significantly younger at seroconversion when compared to children with ICA only (3.4 years and 2.8 years vs 4.8 years) (Table 1 ). Autoantibody profiles at seroconversion varied considerably in the 136 children who developed biochemical islet autoantibodies, both in children who progressed to type 1 diabetes and those who remained autoantibody positive through the follow-up (Table 1). Multiple biochemical autoantibodies were present at seroconversion in $57.8 \%$ of the subjects who progressed to type 1 diabetes, and $95.6 \%$ of progressors became multipositive before diagnosis. The mean age at diagnosis of type 1 diabetes was 8.7 years (SD 4.5; range 1. 9-20.7) among the 45 progressors. All of them carried an HLA risk genotype: $17.8 \%$ had the high risk HLA genotype, $60.0 \%$ moderate risk, and $22.2 \%$ slightly increased HLA genotypes. The children who were at some point positive for 3 to 4 biochemical autoantibodies were younger at diagnosis than those with only 1 to 2 biochemical autoantibodies (mean 7.7 vs 13.2 years, difference 5.5 years, $95 \% \mathrm{Cl} 2.3$ to 8.6 years, $P=.001$ ). There were five adolescents diagnosed after the age of 15 years, and four of them had been GADA positive at seroconversion. One of them had GADA as single biochemical autoantibody through the follow-up (age at diagnosis 20.7 years). The other two tested positive for GADA only at seroconversion (age at diagnosis 17.5 and 16.9 years) but developed multiple autoantibodies during follow-up. The remaining two subjects were multipositive at seroconversion (age at diagnosis 16.1 and 18.4 years).

\subsection{Extended family history of type 1 diabetes}

Structured questionnaires inquiring the family history of diabetes were given to the families at the DIPP visit or sent to the parents by mail. First, the families were asked to fill in the questionnaire. The study nurse contacted the family by phone within 1 to 2 weeks and ensured that all the questions had been understood correctly and answered as completely as possible. The families were systematically asked about the presence of diabetes in the siblings, parents, grandparents, aunts, uncles, cousins, and other relatives of the DIPP child. The type of diabetes (type 1, type 2 or gestational diabetes as reported by the parents), the age at diagnosis, and the mode of treatment (diet, oral medication and/or insulin) were recorded.

The study was approved by the ethics committee of Oulu University Hospital. All participating families provided written informed consent.

\section{5 | Statistical analyses}

The distributions of categorical variables were compared using exact Pearson's chi-squared test. Proportions between two groups were tested by the Standardized Normal Deviate (SND) test. Comparisons of means between two groups were done by Student's $t$ test and between more than two groups by Welch's ANOVA test with GamesHowell procedure for post hoc multiple comparisons correction. Data analysis was performed with the IBM SPSS for Windows (version 25) statistical software (Armonk, NY; IBM Corp.) and StatsDirect statistical software (StatsDirect Ltd, England).

\subsection{PATIENT CONSENT STATEMENT}

All participating families provided written informed consent.

\section{3 | RESULTS}

A total of 214 children (36.8\%) had at least one relative with type 1 diabetes in the extended family or in the nuclear family. We compared the frequency of type 1 diabetes in the families between four groups of children: progressors with biochemical autoantibodies, nonprogressors with biochemical autoantibodies, non-progressors with ICA only, and autoantibody negative children. There were statistically significant differences in the extended family history of type 1 diabetes between children who progressed to type 1 diabetes and those who remained non-diabetic (Table 2). The majority of progressors had at least one relative with type 1 diabetes (62.2\%), whereas in the other groups the proportions were clearly lower. The lowest proportion of children with a relative with type 1 diabetes was observed in the autoantibody negative group (32.4\%). When including only relatives outside the nuclear family the difference between the groups remained statistically significant (Table 2). A similar trend was observed in the nuclear family, particularly when type 1 diabetes in the father was analyzed.

Interestingly, the number of child's biochemical autoantibodies was significantly associated with the presence of type 1 diabetes in any relatives (Tables 3 and 4). Children with 2 to 4 biochemical autoantibodies had more often a positive family history of type 1 diabetes (57.7\%) compared to children with a single biochemical autoantibody (32.3\%) ( $P=.002$; Table 3). Particularly, this effect was present when analyzing the paternal extended family $(32.2 \%$ vs $13.8 \%, P=.010)$. Furthermore, children with multiple biochemical autoantibodies had more often relative(s) with type 1 diabetes outside the nuclear family when compared to those with a single biochemical autoantibody $(P=.006$; Table 3 ). When only nuclear family was considered (Tables $3-4)$, children with multiple autoantibodies had more often a father, mother or sibling with type 1 diabetes than children with single positivity $(P=.019$; Table 3$)$.

Maternal type 1 diabetes was associated with younger seroconversion age of the child when compared to those children without maternal type 1 diabetes (2.0 vs 4.1 years; difference $2.2,95 \% \mathrm{Cl} 1.2$ to $3.2, P=.001)$. Paternal type 1 diabetes was not significantly associated with seroconversion age of the child ( 2.9 vs 4.1 years in children with and without paternal type 1 diabetes, respectively; difference 
TAB LE 2 Family history of type 1 diabetes (T1D) in various groups: autoantibody negative children, autoantibody positive children, children with islet cell antibodies only (ICA only), children with biochemical islet autoantibodies (biochemical Aab) and children who progressed to T1D. Number and percentage of children having at least one relative with T1D is given Comment by Riitta Veijola when reading the proofs: Please correct data lines 6-11 and 14-19 to be located lower to fit with the corresponding text in the left column. e.g. 7 (2.4) should match with T1D in maternal grandparents

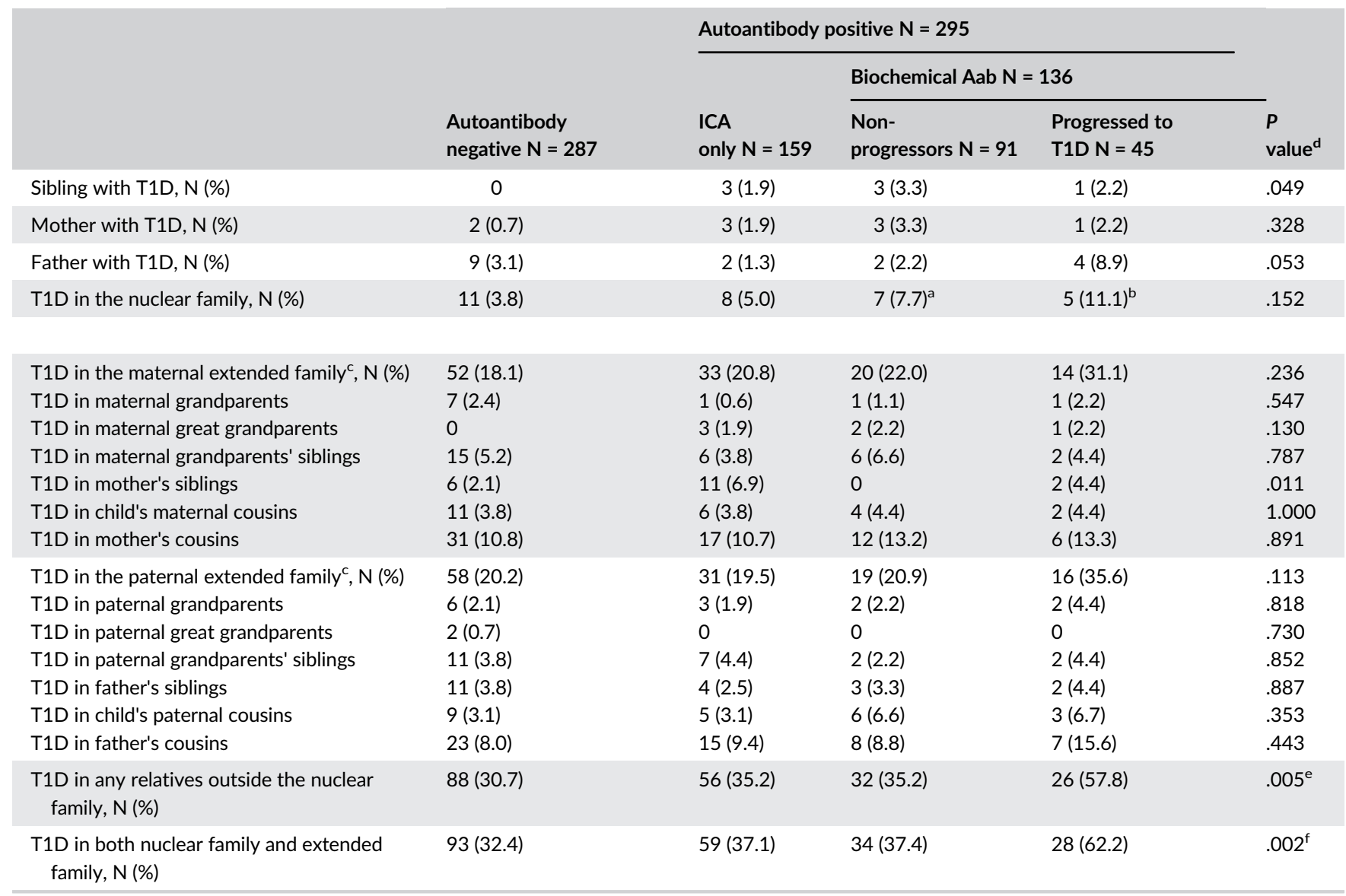

Note: Nuclear family includes full siblings, the mother and the father.

an one family both the father and the sibling had T1D.

${ }^{b}$ In another family both the mother and the father had T1D.

Including the mother or the father.

${ }^{\mathrm{d}}$ Exact Chi square test was used in all comparisons between the four groups.

ePairwise SND tests: Autoantibody negative vs T1D, $P<.001$; ICA only vs T1D, $P=0.006$; Non-progressors vs T1D, $P=0.011$.

${ }^{f}$ Pairwise SND tests: Autoantibody negative vs T1D, $P<.001$; ICA only vs T1D, $P=0.002$; Non-progressors vs T1D, $P=0.007$.

1.2, $95 \% \mathrm{Cl}-1.1$ to $3.4, P=.314)$. In children with multiple islet autoantibodies $(n=71)$ the age at the appearance of multiple antibodies was not affected by family history of type 1 diabetes ( 4.1 years vs 4.6 years in children with and without any relative with type 1 diabetes; $P=.557)$. Neither was the age at initial seroconversion in multipositive children affected by family history (3.1 years for children who had at least one relative with type 1 diabetes and 3.3 years for those who had no relatives with type 1 diabetes; $P=.519$ ).

IAA and GADA were compared as the first appearing islet autoantibody and also as the single biochemical autoantibody during follow-up (Table 5). No differences in the frequency of IAA only or GADA only at seroconversion were observed when comparing children in various family history groups. Neither were any differences seen for IAA only and GADA only during follow-up between these groups.
Other types of diabetes were also reported. In the nuclear families, two mothers and three fathers of autoantibody negative children had type 2 diabetes. In addition, one father of a child with ICA only had type 2 diabetes. However, the 136 children with biochemical autoantibodies had no first-degree relatives with type 2 diabetes. When including all relatives outside the nuclear family, a positive family history of type 2 diabetes was very common in the study children (80.8\%). There were no differences in the extended family history of type 2 diabetes between children without autoantibodies, with ICA only, non-progressors with biochemical autoantibodies and progressors ( $84.0 \%$ vs $76.1 \%$ vs $76.9 \%$ vs $84.4 \%$, respectively).

Seventy-nine mothers (13.6\%) had been diagnosed with gestational diabetes in at least one pregnancy. The proportions of study children with such a mother were similar in the above mentioned four groups (14.3\%, 13.2\%, 14.3\%, and 8.9\%; respectively) 
TAB LE 3 Family history of type 1 diabetes (T1D) in two groups of DIPP children with biochemical autoantibodies according to the maximal number of biochemical autoantibodies during follow-up. Both progressors and non-progressors are included. Number and percentage of children having at least one relative with T1D is given

\begin{tabular}{|c|c|c|c|}
\hline & 1 Biochemical Aab N = 65 & 2-4 Biochemical Aab $N=71$ & $P$ value \\
\hline Sibling with T1D, N (\%) & $1(1.5)$ & $3(4.2)$ & .371 \\
\hline Mother with T1D, N (\%) & $1(1.5)$ & $3(4.2)$ & .371 \\
\hline T1D in the nuclear family, $\mathrm{N}(\%)$ & $2(3.1)$ & $10(14.1)^{b / c}$ & .019 \\
\hline T1D in the maternal extended family ${ }^{\mathrm{a}}, \mathrm{N}(\%)$ & $14(21.5)$ & $20(28.2)$ & .336 \\
\hline T1D in any relatives outside the nuclear family, $\mathrm{N}(\%)$ & $20(30.8)$ & $38(53.5)$ & .006 \\
\hline $\begin{array}{l}\text { T1D in any relatives (nuclear family and extended } \\
\text { family included), } \mathrm{N}(\%)\end{array}$ & $21(32.3)$ & $41(57.7)$ & .002 \\
\hline
\end{tabular}

Note: Nuclear family includes full siblings, the mother and the father. SND test was used in all comparisons.

ancluding the mother or the father.

b In one family both the father and the sibling had T1D.

In one family both the mother and the father had T1D.

TAB LE 4 Family history of type 1 diabetes (T1D) in four groups of DIPP children with biochemical autoantibodies according to the maximal number of biochemical autoantibodies during follow-up. Number and percentage of children having at least one relative with T1D is given

\begin{tabular}{|c|c|c|c|c|c|}
\hline & \multicolumn{5}{|c|}{$\begin{array}{l}\text { Children with Biochemical Aab including both non-progressors and subjects who progressed to } \\
\text { T1D N = } 136\end{array}$} \\
\hline & $\begin{array}{l}1 \text { Biochemical } \\
\text { Aab N = } 65\end{array}$ & $\begin{array}{l}2 \text { Biochemical } \\
\text { Aab } \mathrm{N}=22\end{array}$ & $\begin{array}{l}3 \text { Biochemical } \\
\text { Aab N = } 18\end{array}$ & $\begin{array}{l}4 \text { Biochemical } \\
\text { Aab } N=31\end{array}$ & $P$ value $^{\mathrm{d}}$ \\
\hline Sibling with T1D, N (\%) & $1(1.5)$ & $2(9.1)$ & $1(5.6)$ & 0 & .172 \\
\hline Mother with T1D, N (\%) & $1(1.5)$ & $1(4.5)$ & $1(5.6)$ & $1(3.2)$ & .896 \\
\hline T1D in the maternal extended familyc, $N(\%)$ & $14(21.5)$ & $5(22.7)$ & $6(33.3)$ & $9(29.0)$ & .703 \\
\hline T1D in the paternal extended familyc, $N(\%)$ & 9 (13.8) & $10(45.5)$ & $8(44.4)$ & $8(25.8)$ & $.005^{\mathrm{e}}$ \\
\hline $\begin{array}{l}\text { T1D in any relatives outside the nuclear } \\
\text { family, } \mathrm{N}(\%)\end{array}$ & $20(30.8)$ & $11(50.0)$ & $12(66.7)$ & $15(48.4)$ & $.030^{f}$ \\
\hline
\end{tabular}

Note: Nuclear family includes full siblings, the mother and the father.

an one family both the father and the sibling had T1D.

b In one family both the mother and the father had T1D.

Including the mother or the father.

${ }^{\mathrm{d}}$ Exact Chi square test was used in all comparisons between the four groups.

${ }^{e}$ Pairwise SND tests: 1 Biochemical Aab vs 2 biochemical Aab, $P=0.003 ; 1$ Biochemical Aab vs 3 biochemical Aab, $P=.005$.

fPairwise SND tests: 1 Biochemical Aab vs 3 biochemical Aab, $P=.007$.

${ }^{\mathrm{g}}$ Pairwise SND tests: 1 Biochemical Aab vs 2 biochemical Aab, $P=.026 ; 1$ Biochemical Aab vs 3 biochemical Aab, $P=.008 ; 1$ Biochemical Aab vs 4

biochemical Aab, $P=.053$.

\section{4 | DISCUSSION}

In the prospective DIPP study that follows children with HLA conferred susceptibility from birth, we demonstrate that a positive extended family history of type 1 diabetes is associated with increased risk of islet autoimmunity, the number of biochemical autoantibodies and development of type 1 diabetes. In particular, our data shows for the first time that type 1 diabetes in relatives outside the nuclear family is a significant risk factor for islet autoimmunity and progression to clinical disease in HLA susceptible children.

Earlier studies have reported relatively constant proportions of patients with type 1 diabetes who have first degree relatives with the disease, $10 \%$ to $13 \%$ at the time of diagnosis of the index case. ${ }^{18-21}$ Only very few earlier studies have explored the role of extended family history of type 1 diabetes. In Finland $21.8 \%$ of children with newly diagnosed type 1 diabetes have at least one affected first- and/or 
TAB LE 5 Number of children with biochemical autoantibodies and IAA or GADA as the first autoantibody at seroconversion, or as the only autoantibody during follow-up. Data is shown according to the family history of type 1 diabetes (T1D)

\begin{tabular}{|c|c|c|c|c|c|c|}
\hline & \multicolumn{3}{|l|}{ At seroconversion } & \multicolumn{3}{|c|}{ During the follow-up } \\
\hline Sibling with T1D, N (\%) & $2(4.5)$ & $1(3.0)$ & 0.999 & $1(2.7)$ & $0(0)$ & .999 \\
\hline Mother with T1D, N (\%) & $1(2.2)$ & $1(3.0)$ & 0.999 & $0(0)$ & $1(4.5)$ & .186 \\
\hline Father with T1D, $\mathrm{N}^{5}$ & $1(2.2)$ & $0(0)$ & 0.999 & $0(0)$ & $0(0)$ & \\
\hline T1D in the nuclear family, $\mathrm{N}(\%)$ & $3(6.8)$ & $2(6.1)$ & 0999 & $1(2.7)$ & $1(4.5)$ & .999 \\
\hline T1D in the mother's family, $\mathrm{N}(\%)$ & $12(27.3)$ & $11(33.3)$ & 0.471 & $6(16.2)$ & $8(36.4)$ & .070 \\
\hline T1D in the father's family, $\mathrm{N}$ (\%) & $10(22.7)$ & $4(12.1)$ & 0.252 & $6(16.2)$ & $2(9.1)$ & .467 \\
\hline $\begin{array}{l}\text { T1D in any relative outside the } \\
\text { nuclear family, } \mathrm{N}(\%)\end{array}$ & $18(40.9)$ & $12(36.4)$ & 0.647 & $11(29.7)$ & $8(36.4)$ & .581 \\
\hline
\end{tabular}

Note: SND test was used in all comparisons.

Abbreviations: GADA, antibodies against glutamic acid decarboxylase; IAA, insulin autoantibody; SND, standardized normal deviate.

second-degree relative. In a large series from the Finnish Pediatric Diabetes Register a total of $9.9 \%$ of the cases had affected first degree relative(s), 9.5\% had affected second degree relative(s) (ie, either grandparent or sibling of the parent), and $2.4 \%$ had both first and second degree relatives with type 1 diabetes. ${ }^{22}$ However, third degree relatives (ie, cousins, siblings of grandparents and their children, great grandparents) were not taken into account. In a smaller Finnish series of children with type 1 diabetes $54.0 \%$ of the cases had at least one affected relative when first, second and third degree relatives were included, compared to $32.5 \%$ of matched non-diabetic children. ${ }^{23}$ In the current study population $36.8 \%$ of children initially selected for DIPP follow-up had at least one relative with type 1 diabetes in the whole extended family, and the proportion was remarkably higher (62.2\%) in those who developed biochemical islet autoantibodies and progressed to type 1 diabetes. A surprisingly high proportion of progressors (57.8\%) had an affected relative outside the nuclear family. Among autoantibody negative DIPP children the proportion of those with any relative with type 1 diabetes in the extended family was $32.4 \%$, very similar to the findings by Alhonen et al. in the general childhood population in Finland. ${ }^{23}$ It is noteworthy that the DIPP children represent HLA selected high risk population whereas the Finnish Pediatric Diabetes Register and the study by Alhonen et al. ${ }^{23}$ also included cases with other class II HLA genotypes.

Our novel and the most important finding was that the presence of type 1 diabetes in the extended family was clearly more frequent among the progressors as compared to non-progressors with biochemical autoantibodies, children with ICA only, or those with no detectable autoantibodies; also when considering only relatives outside the nuclear family. Interestingly, the number of various biochemical autoantibodies was significantly associated with a positive family history of type 1 diabetes. Children with single positivity had affected relatives as often as those without any autoantibodies, whereas those with two to four biochemical autoantibodies had positive family history as often as the progressors. Multipositivity was associated with paternal, but not maternal, extended family history of type 1 diabetes, which is a novel observation and of special interest together with the well-known fact that type 1 diabetes in the father confers higher risk for the offspring to develop the disease compared to maternal type 1 diabetes. ${ }^{24}$ Children with either a mother or a father with type 1 diabetes ( $n=7$ and $n=8$, respectively) were slightly younger at seroconversion than other children, although the difference was statistically significant only for maternal type 1 diabetes. The age at appearance of multiple autoantibodies was not affected by family history of type 1 diabetes.

In the group of progressors $43 / 45$ (95.6\%) were positive for multiple biochemical islet autoantibodies and seroconversion age was significantly younger than in the other autoantibody positive children. This is in line with earlier studies reporting that young age at seroconversion is associated with rapid progression to clinical disease. ${ }^{7}$ On the other hand, only $28 / 91$ (30.8\%) of non-progressors with biochemical autoantibodies were multipositive, while 63/91 (69.2\%) tested positive for a single autoantibody. Non-progressors also had less often any family member with type 1 diabetes, and therefore it can be speculated that a lower family burden and thereby perhaps weaker genetic risk protects the child from the development of multiple autoantibodies and the disease. Earlier studies have shown that children with a single autoantibody have remarkably lower risk for development of type 1 diabetes during follow-up for 10 to 15 years compared to those with multiple islet autoantibodies. ${ }^{2}$

The strength of this study lies in the systematically collected extended family history data from families participating in the prospective DIPP study, including also third degree relatives. The followup time of the children was relatively long, more than 10 years in participants who did not progress to type 1 diabetes. Our study 
population included also autoantibody positive children who were followed beyond age 15 years, and a total of five subjects were diagnosed after that age. One major asset was that we collected family history data also from DIPP children remaining autoantibody negative and both groups were followed in parallel.

A limitation was that even though the family history data were based on both structured questionnaires and interviews by a trained nurse, the parents may have had inaccurate or inadequate knowledge about disease history in the extended family. We only recorded the diagnoses that the parents considered reliable. However, in some cases there might have been misclassification between type 1 and type 2 diabetes. In the current series we were not able to explore the relationship between family history of type 1 diabetes and class II HLA genotype, because the DIPP study is focused on follow-up of children with increased HLA-conferred risk.

Type 1 diabetes is currently not preventable, but our knowledge about predictive markers has greatly improved with the data from prospective birth cohort studies. ${ }^{5,6}$ In addition, type 1 diabetes genetic risk scores (GRS) which have recently been developed have been shown to improve prediction of the disease. ${ }^{25}$ We anticipate that in the future the combination of population specific GRS and extended family history could still improve the prediction of islet autoimmunity and type 1 diabetes.

In conclusion, our data demonstrates that in addition to type 1 diabetes in the nuclear family, the presence of the disease in relatives outside the nuclear family, particularly in the paternal family, is associated with development of multiple islet autoantibodies and progression to type 1 diabetes in children at genetic risk. Future studies should include collection of data from the extended family of subjects at risk for type 1 diabetes.

\section{ACKNOWLEDGEMENTS}

All families participating in the DIPP study are acknowledged for their valuable contribution. Authors thank also the personnel in the study clinics and in the genetic and autoantibody laboratories for their contribution to the success of the DIPP study.

\section{CONFLICT OF INTEREST}

The authors declare no potential conflict of interest.

\section{AUTHOR CONTRIBUTIONS}

Salla Kuusela, Riitta Veijola and Tytti Pokka performed the research and analyzed the data. Salla Kuusela, Riitta Veijola, Tytti Pokka and Päivi Keskinen drafted the manuscript. All authors participated in revising the manuscript and approved the final version.

\section{DATA AVAILABILITY}

The data generated and analyzed during the current study are available from the corresponding author upon reasonable request.

\section{ETHICS STATEMENT}

The study was approved by the ethics committee of Oulu University Hospital.

\section{PEER REVIEW}

The peer review history for this article is available at https://publons. com/publon/10.1111/pedi.13122.

\section{ORCID}

Salla Kuusela (D) https://orcid.org/0000-0003-4945-3089

Mikael Knip (DD https://orcid.org/0000-0003-0474-0033

Riitta Veijola (D) https://orcid.org/0000-0002-6557-270X

\section{REFERENCES}

1. Harjutsalo V, Sjöberg L, Tuomilehto J. Time trends in the incidence of type 1 diabetes in Finnish children: a cohort study. Lancet. 2008;371 (9626):1777-1782. https://doi.org/10.1016/S0140-6736.

2. Ziegler AG, Rewers $M$, Simell $O$, et al. Seroconversion to multiple islet autoantibodies and risk of progression to diabetes in children. Jama. 2013;19(23):2473-2479. https://doi.org/10.1001/jama.2013.6285.

3. Atkinson MA, Eisenbarth GS. Type 1 diabetes: new perspectives on disease pathogenesis and treatment. Lancet. 2001;358(9277):221229. https://doi.org/10.1016/S0140-6736.

4. Ziegler AG, Bonifacio E, BABYDIAB-BABYDIET Study Group. Agerelated islet autoantibody incidence in offspring of patients with type 1 diabetes. Diabetologia. 2012;55(7):1937-1943. https://doi.org/10. 1007/s00125-012-2472-x.

5. Ilonen J, Hammais A, Laine AP, et al. Patterns of beta-cell autoantibody appearance and genetic associations during the first years of life. Diabetes. 2013;62(10):3636-3640. https://doi.org/10.2337/ db13-0300.

6. Krischer JP, Lynch KF, Schatz DA, et al. The 6 year incidence of diabetes-associated autoantibodies in genetically at-risk children: the TEDDY study. Diabetologia. 2015;58(5):980-987. https://doi.org/10. 1007/s00125-015-3514-y.

7. Parikka V, Näntö-Salonen K, Saarinen M, et al. Early seroconversion and rapidly increasing autoantibody concentrations predict prepubertal manifestation of type 1 diabetes in children at genetic risk. Diabetologia. 2012;55(7):1926-1936. https://doi.org/10.1007/ s00125-012-2523-3.

8. Knip M, Siljander H, llonen J, Simell O, Veijola R. Role of humoral beta-cell autoimmunity in type 1 diabetes. Pediatr Diabetes. 2016;17 (22):17-24. https://doi.org/10.1111/pedi.12386.

9. Pöllänen $P M$, Lempainen J, Laine $A P$, et al. Characterisation of rapid progressors to type 1 diabetes among children with HLA-conferred disease susceptibility. Diabetologia. 2017;60(7):1284-1293. https:// doi.org/10.1007/s00125-017-4258-7.

10. Pöllänen $P M$, Lempainen J, Laine $A P$, et al. Characteristics of slow progression to type 1 diabetes in children with increased HLAconferred disease risk. J Clin Endocrinol Metab. 2017;17:5585-5594. https://doi.org/10.1210/jc.2019-01069.

11. Harjutsalo V, Reunanen A, Tuomilehto J. Differential transmission of type 1 diabetes from diabetic fathers and mothers to their offspring. Diabetes. 2006;55(5):1517-1524. https://doi.org/10.2337/db051296.

12. Warram JH, Krolewski AS, Gottlieb MS, Kahn CR. Differences in risk of insulin-dependent diabetes in offspring of diabetic mothers and diabetic fathers. N Engl J Med. 1984;311(3):149-152. https://doi.org/ 10.1056/NEJM198407193110304.

13. Lebenthal Y, de Vries L, Phillip M, Lazar L. Familial type 1 diabetes mellitus - gender distribution and age at onset of diabetes distinguish between parent-offspring and sib-pair subgroups. Pediatr Diabetes. 2010;11(6):403-411. https://doi.org/10.1111/j.1399-5448.2009. 00621.x.

14. Kukko $M$, Kimpimäki $T$, Kupila $A$, et al. Signs of beta-cell autoimmunity and HLA-defined diabetes susceptibility in the Finnish 
population: the sib cohort from the type 1 diabetes prediction and prevention study. Diabetologia. 2003;46(1):65-70. https://doi.org/10. 1007/s00125-002-0976-5.

15. Aly TA, Ide A, Jahromi MM, et al. Extreme genetic risk for type $1 \mathrm{~A}$ diabetes. Proc Natl Acad Sci U S A. 2006;103(38):14074-14079. https://doi.org/10.1073/pnas.0606349103.

16. Redondo MJ, Oram RA, Steck AK. Genetic risk scores for type 1 diabetes prediction and diagnosis. Curr Diab Rep. 2017;17(12):129. https://doi.org/10.1007/s11892-017-0961-5.

17. Ilonen J, Kiviniemi M, Lempainen J, et al. Genetic susceptibility to type 1 diabetes in childhood - estimation of HLA class II associated disease risk and class II effect in various phases of islet autoimmunity. Pediatr Diabetes. 2016;17(22):8-16. https://doi.org/10.1111/pedi. 12327.

18. Dahlquist G, Blom L, Holmgren G, et al. The epidemiology of diabetes in Swedish children 0-14 years-a six-year prospective study. Diabetologia. 1985;28(11):802-808. https://doi.org/10.1007/BFO 0291068.

19. Pociot F, Norgaard K, Hobolth N, Andersen O, Nerup J. A nationwide population-based study of the familial aggregation of type 1 (insulindependent) diabetes mellitus in Denmark, Danish Study Group of Diabetes in Childhood. Diabetologia. 1993;36(9):870-875. https://doi. org/10.1007/BF00400364.

20. Veijola R, Reijonen H, Vähäsalo $P$, et al. HLA-DQB1-defined genetic susceptibility, beta cell autoimmunity, and metabolic characteristics in familial and nonfamilial insulin-dependent diabetes mellitus. Childhood diabetes in Finland (DiMe) study group. J Clin Invest. 1996;98(11):2489-2495. https://doi.org/10.1172/ JCl119067.
21. Turtinen M, Härkönen T, Parkkola A, Ilonen J, Knip M, Finnish Pediatric Diabetes Register. Characteristics of familial type 1 diabetes: effects of the relationship to the affected family member on phenotype and genotype at diagnosis. Diabetologia. 2019;62(11):20252039. https://doi.org/10.1007/s00125-019-4952-8.

22. Parkkola A, Härkönen T, Ryhänen SJ, Ilonen J, Knip M, Finnish Pediatric Diabetes Register. Extended family history of type 1 diabetes and phenotype and genotype of newly diagnosed children. Diabetes Care. 2013;36(2):348-354. https://doi.org/10.2337/dc12-0445.

23. Alhonen S, Korhonen S, Tapanainen P, Knip M, Veijola R. Extended family history of diabetes and autoimmune diseases in children with and without type 1 diabetes. Diabetes Care. 2011;34(1):115-117. https://doi.org/10.2337/dc10-1091.

24. Gale EA, Gillespie KM. Diabetes and gender. Diabetologia. 2001;44(1): 3-15. https://doi.org/10.1007/s001250051573.

25. Sharp SA, Rich SS, Wood AR, et al. Development and standardization of an improved type 1 diabetes genetic risk score for use in newborn screening and incident diagnosis. Diabetes Care. 2019;42(2):200-207. https://doi.org/10.2337/dc18-1785.

How to cite this article: Kuusela S, Keskinen P, Pokka T, et al. Extended family history of type 1 diabetes in HLApredisposed children with and without islet autoantibodies. Pediatr Diabetes. 2020;21:1447-1456. https://doi.org/10. 1111/pedi.13122 